\title{
Quantum Structures of the Hydrogen Atom
}

\author{
Jasmina Jeknić-Dugić ${ }^{*}$, Miroljub Dugić ${ }^{2}$, Allen Francom³ ${ }^{3}$ Momir Arsenijevićc ${ }^{2}$ \\ ${ }^{1}$ Department of Physics, Faculty of Science, Niš, Serbia \\ ${ }^{2}$ Department of Physics, Faculty of Science, Kragujevac, Serbia \\ ${ }^{3}$ Indpendent Author, Austin, USA \\ Email: ${ }^{*}$ jieknic@pmf.ni.ac.rs
}

Received 7 April 2014; revised 23 May 2014; accepted 19 June 2014

Copyright (C) 2014 by authors and OALib.

This work is licensed under the Creative Commons Attribution International License (CC BY).

http://creativecommons.org/licenses/by/4.0/

\section{(c) (i) Open Access}

\begin{abstract}
Modern quantum theory introduces quantum structures (decompositions into subsystems) as a new discourse that is not fully comparable with the classical-physics counterpart. To this end, socalled Entanglement Relativity appears as a corollary of the universally valid quantum mechanics that can provide for a deeper and more elaborate description of the composite quantum systems. In this paper we employ this new concept to describe the hydrogen atom. We offer a consistent picture of the hydrogen atom as an open quantum system that naturally answers the following important questions: 1) how do the so called "quantum jumps" in atomic excitation and de-excitation occur? and 2) why does the classically and seemingly artificial "center-of-mass + relative degrees of freedom" structure appear as the primarily operable form in most of the experimental reality of atoms?
\end{abstract}

\section{Keywords}

Hydrogen Atom, Quantum Correlations, Open Quantum Systems

Subject Areas: Quantum Mechanics, Theoretical Physics

\section{Introduction}

W. H. Zurek [1] presents a criticism of the anachronistic teaching approach of quantum mechanics which is that it appears to be a disservice to the ideology required by modern degrees of understanding.

"Quantum mechanics has been to date, by and large, presented in a manner that reflects its historical development. That is, Bohr's planetary model of the atom is still often the point of departure, Hamilton-Jacobi equations are used to "derive" the Schrödinger equation, and an oversimplified version of the quantum-classical relationship (attributed to Bohr, but generally not doing justice to his much more sophisticated views) with the correspondence principle, kinship of commutators and Poisson brackets, the Ehrenfest theorem, some version of

${ }^{*}$ Corresponding author. 
the Copenhagen interpretation, and other evidence that quantum theory is really not all that different from classical-especially when systems of interest become macroscopic, and all one cares about are averages-is presented".

This paper can be considered an attempt to remedy this shared criticism by providing a more modern introduction that is less encumbered by classical reasoning.

So to begin, the hydrogen atom is among the most and best investigated of all physical systems serving as the paradigmatic foundation of non-relativistic quantum physics and thus a wide range of scientific and technological understanding. Today the science and theory have evolved and we are more interested in the different structures also called subsystems of the Hydrogen Atom, such as the Electron and Proton $(e+p)$, as one kind decomposition, and the Center-of-Mass + Relative Position $(\mathrm{CM}+\mathrm{R})$ as another kind of decomposition.

At first glance, the Electron and Proton are real things, and the Center-of-Mass and Relative Position are abstract ideas. To the modern view these structural decompositions are each no less real than the other. In classical physics, such structural or subsystem considerations are generally considered artificial, that is to say that a composite system's center-of-mass is usually an empty point in space, not a physical object that can be observed or realistically targeted in an experiment. However, in contrast to this classical mindset, atomic physics phenomenology directly considers this $\mathrm{CM}+\mathrm{R}$ atomic structure. This contrast is typically ignored by virtually identifying the atomic e and R systems on the one hand, and the p and CM systems on the other hand, cf. Section 2 for details.

Entanglement Relativity [2]-[10] is an important, subtle, and as yet often overlooked part of modern quantum theory which lends itself directly to the clarification of these issues with structures. Therefore it is on the basis of Entanglement Relativity [2]-[10] in Section 3 that we explore and highlight these crucial subtleties in distinguishing between the two above mentioned structures of the Hydrogen Atom and provide what we consider to be a more modern and proper description of experimental reality. By considering the Hydrogen Atom as an open [11]-[14] quantum system in Section 4, we answer the following important questions: 1) how do the so called "quantum jumps" in atomic excitation and de-excitation occur? and 2) why does the classical CM + R structure appear as the primarily operable form in most of the realistic experimental situations with atoms in spite of its seemingly artificial nature. Section 5 summarizes the answers obtained in a more general form. Section 6 is a Discussion and we conclude in Section 7.

\section{The Hydrogen Atom: The Standard View}

Historically, it was the observation of discrete energy spectra for atoms of the different chemical elements which has been at the core of the development of quantum theory. Physically, these observations come from both the spectroscopic results as well as from the inelastic collisions of the atoms with, for example, electrons.

Detailed observations of the inelastic collisions between atoms and electrons, and more precisely the loss of the total kinetic energy in such collisions reveals that the internal energy of atoms appears to be discrete, or "quantized", gaining or losing only whole integer values of energy. This is in sharp contrast with the macroscopic world we live in wherein it is universally observed that classical macroscopic bodies like billiard balls can accelerate or be accelerated smoothly, that is to say with a continuous energy spectra that is not quantized into discrete integer-like jumps from one speed to another. Further investigations into the structure of matter has led to the conclusion that the hydrogen atom is not a fundamental particle itself but is instead a composition of a single electron and a single proton $(e+p)$, and this is now considered as the fundamental non-relativistic model of the Hydrogen Atom.

In keeping with a classical-physics model such as the "Solar System Model" for atoms, the atomic internal degrees of freedom refer usually to the atomic electrons (e). This then has served as the basis for commonly used thoughts and terms such as "electron orbits" and "electron energies" in describing the internal structure and internal energy of the atomic elements. However, this is imprecise and as we emphasize in Section 5, physically naive.

\subsection{The Textbook Theoretical Model of the Hydrogen Atom}

Bearing in mind the fundamental definition e + p, the Hydrogen Atom is defined by way of the following Hamiltonian where the model is non-relativistic and spin is not considered 


$$
\hat{H}=\hat{T}_{e}+\hat{T}_{p}+\hat{V}_{\text {Coul }}=\frac{\hat{\mathbf{p}}_{e}^{2}}{2 m_{e}}+\frac{\hat{\mathbf{p}}_{p}^{2}}{2 m_{p}}-k \frac{e^{2}}{\left|\hat{\mathbf{r}}_{e}-\hat{\mathbf{r}}_{p}\right|}
$$

In Equation (1): $k$ is the standard Coulomb constant, $k=\left(4 \pi \varepsilon_{0}\right)^{-1}$, and the indices e and p refer to the electron and the proton, respectively.

However, Equation (1) is not really the standard quantum-mechanical theory of the Hydrogen Atom. In keeping with the formalism of classical mechanics, what is considered standard here is the alternative form with separation of variables. These new variables simplify the calculation as well as present an alternative description:

$$
\hat{H}=\hat{T}_{C M}+\hat{H}_{R}=\frac{\hat{\mathbf{p}}_{C M}^{2}}{2 M}+\frac{\hat{\mathbf{p}}_{R}}{2 \mu_{R}}-k \frac{e^{2}}{\left|\hat{\rho}_{R}\right|} ; M=m_{e}+m_{p}, \mu_{R}=\left(m_{e}^{-1}+m_{p}^{-1}\right)^{-1}
$$

In Equation (2), the alternate degrees of freedom are the atomic center-of-mass (CM) and the "relative position” (R), and their formal presentation is given in Equation (5) below. It follows from Equation (2), that the formal systems CM and R simplify the model by separating the variables, and there is not any coupling between the $\mathrm{CM}$ and $\mathrm{R}$ variables.

Physically, the degrees of freedom provided by CM describe the atom as a whole, very much like the classically Newtonian or "ballistic" description. The internal degrees of freedom and the related internal atomic energies refer to the atomic system R. Therefore and thereby we can present the solution of the Schrödinger equation for $\mathrm{R}$ in a form that is uncoupled from the Schrödinger equation for CM:

$$
\hat{H}_{R}\left|n l m_{l}\right\rangle_{R}=E_{n}\left|n l m_{l}\right\rangle_{R}
$$

Equation (3) above gives both the eigenvalues $\left(E_{n}\right)$ and the eigenstates $\left(\left|n l m_{l}\right\rangle_{R}\right)$ of the R's Hamiltonian $\hat{H}_{R} ; n$ is the energy (the "principal") quantum number, while $l$ is the angular-momentum- and $m_{l}$ the magneticquantum number. This resolves the classically paradoxical discrete energy spectra for the internal degrees of freedom for the chemical elements. Every value of the principal quantum number $n=1,2,3, \cdots$ defines one possible internal energy of the atom. By taking the spin into consideration (in the perturbative manner), the other quantum numbers can also contribute to defining the possible internal energies of the atom, while bearing in mind their well-known relations, $l=1,2,3, \cdots, n-1$ and $m=-l,-l+1, \cdots, 0, \cdots, l-1, l$. In the position-representation, the internal-energy $\left(\hat{H}_{R}\right)$ eigenstates $\left|n l m_{l}\right\rangle_{R}$ obtain the familiar form of the "wave-function", $\psi_{n l m_{l}}\left(\rho_{R}\right)$.

Most tasks [15] that we are generally faced with while working with the atoms deal with the seemingly nonfundamental and seemingly abstract CM + R structure. For example, atomic and molecular interferometry and manipulation rely heavily on the CM system, while in atomic spectroscopy we refer fairly exclusively to the R system. And so we might say that our everyday experience and phenomenology rely practically and almost exclusively on the $\mathrm{CM}+\mathrm{R}$ structure and form.

\subsection{The Basic Phenomenological Rule of Atomic Physics}

The success of atomic physics is intimately linked to the following phenomenological rule:

An isolated atom spontaneously decays by making a transition from an excited state with higher energy $E_{m}$ into a lower energy-state $E_{n}$ by emitting a photon of the frequency $v=\left(E_{m}-E_{n}\right) / h$, where $h$ is the Planck's constant.

These transitions which ultimately lead to the only stable state, the so called "ground-energy state", are commonly described in terms using phrases such as "Quantum Jumps”. "Quantum Jumps" is a deprecated form of phrasing and reasoning that has proven incompatible with the Schrödinger Law.

As it is well known, Equation (3) is equivalent with the time evolution governed by the unitary operator $\hat{U}(t)=\exp \left(-l t \hat{H}_{R} / \hbar\right)$. Then the probability of transition $\left|n l m_{l}\right\rangle_{R} \rightarrow\left|n^{\prime} l^{\prime} m_{l^{\prime}}^{\prime}\right\rangle_{R},\left|{ }_{R}\left\langle n^{\prime} l^{\prime} m_{l^{\prime}}^{\prime}|\hat{U}(t)| n l m_{l}\right\rangle_{R}\right|^{2}=0$ in every instant in time, $t$, unless $n=n^{\prime}, l=l^{\prime}, m_{l}=m_{l^{\prime}}^{\prime}$. Physically, "Quantum Jumps" require intervention of an external field or system on the atom. The way out of this apparent contradiction is offered by modern theory of open quantum systems, as we express in Section 4.

In the next section we emphasize some subtleties regarding the Hydrogen Atom's structure that prepare for 
the analysis presented in Section 4.

\section{Quantum Structures}

Symbolically, the Hydrogen Atom (HA) can be presented as: e + p = HA = CM + R. In the quantum-mechanical formalism, it means that the HA Hilbert state space, $H$, can be factorized as $H_{e} \otimes H_{p}$ likewise as $H_{C M} \otimes H_{R}$. Of course, $H_{e} \otimes H_{p}=H=H_{C M} \otimes H_{R}$. Bearing this in mind, the HA Hamiltonian, $\hat{H}$, Equations (1), (2), can be presented precisely as:

$$
\hat{T}_{e} \otimes \hat{I}_{p}+\hat{I}_{e} \otimes \hat{T}_{p}+\hat{V}_{\text {Coul }}=\hat{H}=\hat{T}_{C M} \otimes \hat{I}_{R}+\hat{I}_{C M} \otimes \hat{H}_{R}
$$

In Equation (4) appear the identity operators $\hat{I}$ for the respective factor-spaces of $H$.

The two structures of the Hydrogen Atom, $\mathrm{e}+\mathrm{p}$ and $\mathrm{CM}+\mathrm{R}$, are mutually linked by the well-known linear (and therefore invertible) canonical transformations (LCT) that introduce CM and R as follows:

$$
\hat{\mathbf{R}}_{C M}=\frac{m_{e} \hat{\mathbf{r}}_{e}+m_{p} \hat{\mathbf{r}}_{p}}{m_{e}+m_{p}}, \hat{\rho}_{R}=\hat{\mathbf{r}}_{e}-\hat{\mathbf{r}}_{p}
$$

It is a classical and not quantum mechanical reasoning which states that the Linear Canonical Transformations can serve only as a mathematical tool or that they exist as a mathematical artifact, and not yet as a physically relevant method referring in any way to the physical reality of physical objects. Indeed, a pair of apples' center-of-mass is an empty point in space, not an object. However, this classical statement is in sharp contrast to the core of atomic phenomenology, which refers mainly to the seemingly non-fundamental atomic structure CM $+\mathrm{R}$. Resolving this apparent conflict requires the careful and thoughtful analysis presented in the remainder of this paper.

\subsection{Entanglement in the Hydrogen Atom}

Entanglement Relativity (ER) is a recently established quantum-mechanical rule. As a corollary of quantum mechanics, ER establishes [2]-[10]:

(ER) Typically, a separable form of a pure quantum state $|\Psi\rangle$ for one decomposition obtains the entangled form for another decomposition of a composite system.

In more picturesque terms, there is entanglement for every quantum state of a composite system as this follows from kinematics and dynamics. Given these considerations, the model of the Hydrogen Atom serves as paradigmatic.

Kinematic arguments. Consider the product of two functions $f(x) g(y)$ of two independent variables, $x$ and $y$. If we introduce new variables defined as $\xi=(x+y) / 2$ and $\eta=x-y$, then equality applies,

$f(x) g(y)=f((2 \xi+\eta) / 2) g((2 \xi-\eta) / 2)$. The point is that typically the new functions $F(\xi)$ and $G(\eta)$ do not exist, such that $f(x) g(y)=F(\xi) G(\eta)$.

Getting back to the Hydrogen Atom, the atomic instantaneous state for the CM + R decomposition is of the separable tensor-product form $|\chi\rangle_{C M}\left|n \operatorname{lm}_{l}\right\rangle_{R}$. In the position representation this form is of the type considered above, $f(x) g(y)$ (cf. also Equation (7)). Now, introducing the e and p degrees of freedom (the inverse to Equation (5)), this separability of the state's form is lost-the quantum state for the e $+\mathrm{p}$ decomposition becomes entangled, that is, of the form $\sum_{i} c_{i}\left|\phi_{i}\right\rangle_{e}\left|\varphi_{i}\right\rangle_{p}$.

The rigorous proof of this finding can be found in the literature e.g. [6] [10].

Dynamic arguments. Interacting quantum systems are always entangled. So it is with the atomic e $+p$ structure. Actually, the Coulomb interaction in Equation (1) entangles the hydrogen-atom's electron and proton. In an instant of time, the atomic state for the $\mathrm{e}+\mathrm{p}$ structure obtains the entangled form, $\sum_{i} c_{i}\left|\phi_{i}\right\rangle_{e}\left|\varphi_{i}\right\rangle_{p}$. On the other hand, due to non-interaction of the atomic $\mathrm{CM}$ and $\mathrm{R}$ systems for the isolated atom, the Schrödinger dynamics for the atom as a whole preserves the tensor-product form $|\chi\rangle_{C M}\left|n l m_{l}\right\rangle_{R}$ for every instant of time. So, the Schrödinger dynamics for the hydrogen atom as a whole simultaneously preserves separability of states for CM $+\mathrm{R}$ and induces entanglement for $\mathrm{e}+\mathrm{p}$ structure. A dynamical proof of the presence of entanglement for the e + p structure can be found in the literature [16].

In summary so far, we emphasize that as the universally valid quantum mechanics establishes a unique quan- 
tum state in every instant in time, there is the following equality stemming from both kinematic and dynamic considerations for the Hydrogen Atom:

$$
|\chi\rangle_{C M} \otimes\left|n m_{l}\right\rangle_{R}=\sum_{i} c_{i}\left|\phi_{i}\right\rangle_{e} \otimes\left|\varphi_{i}\right\rangle_{p}
$$

In the position-representation Equation (6) reads:

$$
\chi\left(\mathbf{R}_{C M}\right) \psi_{n l m}\left(\boldsymbol{\rho}_{R}\right)=\sum_{i} c_{i} \phi_{i}\left(\hat{\mathbf{r}}_{e}\right) \varphi_{i}\left(\hat{\mathbf{r}}_{p}\right)
$$

While the standard theory (Section 2) focuses on the left hand side of Equation (7), that is to say it deals with the "wave functions" $\chi\left(\mathbf{R}_{C M}\right)$ and $\psi_{n l m_{l}}\left(\rho_{R}\right)$, modern quantum mechanics extends this standard picture.

\subsection{Manipulating the Atomic Degrees of Freedom}

Like the concept of entanglement in Section 3.1, the concept of local system is also relative [3] [5]. For the hydrogen atom, the "electron" e and the measurements of its variables are local only for the e + p structure. Regarding the alternative $\mathrm{CM}+\mathrm{R}$ structure, the measurements performed on e are partially composite, or in other words "collective". To see this, just invert the expressions in Equation (5):

$$
\hat{\mathbf{r}}_{e}=\hat{\mathbf{R}}_{C M}+\frac{m_{p} \hat{\rho}_{R}}{M}, \quad \hat{\mathbf{r}}_{p}=\hat{\mathbf{R}}_{C M}-\frac{m_{e} \hat{\boldsymbol{\rho}}_{R}}{M}
$$

So for example, a measurement of the electron's position $\hat{\mathbf{r}}_{e}$ is partially a measurement of positions of both $\mathrm{CM}$ and R. The "total" measurement of the positions of both CM and R reveals the values of the positions of both $e$ and $p$. This relativity of local system is a general feature of the composite system's structures. Formally, locality of a subsystem is distinguished by the appearance of the identity operator, $\hat{I}$, for a given structure. For example, for the electron's position observable, $\hat{\mathbf{r}}_{e} \otimes \hat{I}_{p}$, or for the CM energy, $\hat{H}_{C M} \otimes l_{R}$, Equation (6) and Equation (7), respectively.

To illustrate the relativity of "structure" (local system) from the operational perspective, we briefly examine some typical experimental situations not only with atoms.

- Atomic de-excitation. As it is emphasized in Section 3.2, it is a general spectroscopic fact that the higher (internal) atomic energy quickly decays and is accompanied by emission of a photon of some frequency $v$. The frequencies of the emitted photons are characteristic for every chemical element. Operationally, the decay is "directly" observed by observing the emitted photon and by measuring its frequency. Now, bearing in mind the different structures of the Hydrogen Atom, i.e. Section 3.1, we can say: Observation of the photon reveals that the R state change (decay) and (in idealized situation) represents indirect quantum measurement of the R's initial energy. For such physical situations, the "fundamental" structure, $\mathrm{e}+\mathrm{p}$, is of no use-the local subsystem of the Hydrogen Atom that is of interest is R and its quantum state $\psi_{n l m_{l}}\left(\boldsymbol{\rho}_{R}\right)$, not the electron e and its states $\phi_{i}\left(\mathbf{r}_{e}\right)$, Equation (7).

- Interference experiments. In past decades, atomic interferometry has attracted much attention, for one example, by releasing a cloud of metastable atoms towards a screen with the two open slits (an analogue of the famous Young two-slit experiment in optics), one can observe the interference fringes (for a review see e.g. Section 6 in Ref. [15]) - the points of the impact of the individual atoms on the final screen. Clearly, these spots reveal the atomic CM's position on the final screen without making any intervention between the atomic source and the final screen. Certainly, the local subsystem of interest is the atomic CM system, not the atomic nucleus.

- The Stern-Gerlach-like experiments and generalizations. Application of some external field usually couples external degrees of freedom (e.g. the atomic CM system) with the internal degrees of freedom, such as the atomic spin in the Stern-Gerlach experiment. If the external field(s) also couple the CM and R systems, one can design new procedures for manipulating the CM dynamic control. For example, in the Stern-Gerlachlike experiments with molecules [17] [18], this opportunity is very fruitful. Again, the operationally preferred structure is $\mathrm{CM}+\mathrm{R}$.

- Laser cooling of atoms. The remarkable experiments reveal the possibility to cool down an atomic gas, see e.g. Section 7 in Ref. [15]. By properly applying a laser field to the gas, one can induce the internal atomic (the R's) state transitions depending on the atomic velocity (on the atomic CM velocity). So, by externally 
(partially) controlling the laser-light absorption and the photon emission, one can manipulate with the atomic CM kinetic energy (velocity). In effect, one can obtain lower temperature of the gas. Again, the structure of interest is the atomic $\mathrm{CM}+\mathrm{R}$ structure.

- The semi-classical atomic orbits. The proper action exerted on excited lithium atoms can have the effect of producing "Kepler-like orbits" in the lithium atoms [19]. In the manner described in Section 2, these "orbits" are described as the "electron's orbits". For a very short interval of time, the atom resembles the classical Rutherford atom resembling a solar system. However, in the theoretical support and explanation of the experiment, the use of the R's wave functions, $\psi_{n l m_{l}}\left(\rho_{R}\right)$, not of the electron's states, $\phi_{i}\left(\mathbf{r}_{e}\right)$, is made. So, borrowing the notation for HA: the preferred structure of the atom in this situation is $\mathrm{CM}+\mathrm{R}$, not e $+\mathrm{p}$. This experiment is a remarkable confirmation of the more general theoretical considerations [20] of the direct accessibility of the R system. Only in classical-physics terms, i.e. in visualization analogous to the Solar-system's composition, may one express the effect in terms of the atomic electron. While this supports intuition, it is, strictly speaking, not physically correct [20].

- Investigating the structure of the matter. As in the epoch Rutherford's experiment, one can target the composite systems by energetic quantum particles or fields in order to observe the deeper spatial structure of a composite system. As to the atomic species, ionizing the atoms reveals the presence of the electrons, while bombarding the atoms by the energetic particles may reveal the atomic nucleus. So, in these physical situations, the preferred structure is e $+\mathrm{p}$ and e and $\mathrm{p}$ appear as "directly" accessible systems local to this structure.

From the above considerations, we learn: every physical situation distinguishes a preferred structure of a composite system and the related "directly observable” local systems.

\subsection{Equality and Non-Equivalence of the Structures}

For an isolated quantum system, the universally valid quantum mechanics does not a priori set a privileged structure. By Zanardi’s [2]:

"Without further physical assumption, no partition has an ontologically superior status with respect to any other".

This is a direct consequence of the universally valid quantum mechanics. A composite system's Hamiltonian is unique and the system's quantum state is also unique in every instant in time. The state typically takes different forms for different structures, Section 3.1. But the general rules and logic for describing the subsystems are the same for every structure.

This democratic view to the HA structures is not applicable anymore, regarding the predictions for the two structures. In this sense, the two structures are not mutually equivalent. E.g., complete knowledge of the electron's state is in no sense sufficient for description of the atomic CM or R systems, and vice versa. The wave functions for e, $\phi_{i}\left(\mathbf{r}_{e}\right)$, and $\mathrm{R}, \psi_{n l m_{l}}\left(\boldsymbol{\rho}_{R}\right)$ cannot be even compared to each other; and analogously for the $\mathrm{p}$ and CM subsystems. Mathematically, they belong to different "probability spaces", e.g., the integration $\int \mid \psi\left(\rho_{R}\right)^{2} \mathrm{~d}^{3} \hat{\mathbf{r}}_{p}$ does not provide the probability density for e. As emphasized above, only the state of the atom as a whole provides the probability density for arbitrary observable of the atom, i.e. of any of the structures.

These subtle notions equally refer to the possible structures of arbitrary composite quantum systems. To this end, the hydrogen atom, as the simplest possible composite system, is paradigmatic.

\section{Hydrogen Atom Is an Open Quantum System}

As emphasized in Section 2.2, the phenomenological fact that the "excited" internal-energy states are not stable clearly demonstrates that the model of the isolated hydrogen atom, Equations (1)-(3), is not correct or is at least not complete.

The proper physical picture offered in modern quantum theory sticks to the later. The hydrogen atom is assumed to interact with another physical system, the so called quantum vacuum fluctuations (QVF). The total system "Atom + QVF" is now supposed to be described by the Schrödinger Law which makes the atom non-describable by the Schrödinger Law. The atom is then said to be "open" [11]. The quantum vacuum fluctuation system effectively monitors the internal atomic (R) state and provides the smooth (unitary and even time-reversible) state change of the total system "Atom + QVF". With emission of one photon, the state change reads, e.g.: 


$$
\left|n=2, l=0, m_{l}=0\right\rangle_{R} \otimes|0\rangle_{Q V F} \rightarrow\left|n=1, l=0, m_{l}=0\right\rangle_{R} \otimes|1\rangle_{Q V F}
$$

Now, by ignoring the QVF system in Equation (9), one obtains impression of the "quantum jumps" of Section 2.2: $\left|n=2, l=0, m_{l}=0\right\rangle_{R} \rightarrow\left|n=1, l=0, m_{l}=0\right\rangle_{R}$.

\subsection{Arguments from the Theory of Open Quantum Systems}

From Equation (9) and from Section 3.3, we learn: the environment QVF targets only the atomic R-system and thus makes a choice of the preferred structure $\mathrm{CM}+\mathrm{R}$ of the atom. Only for the $\mathrm{CM}+\mathrm{R}$ structure the external influence of QVF is local, and not for e + p.

This is exactly the structure that is both "directly" accessible (as described in Section 3.2) as well as usually described in the quantum mechanics textbooks (cf. Section 2).

The related mathematical details can be found in the literature e.g. [11] [21] [22]. These considerations are open to further technical improvements and there are also some open issues related to interpretation (e.g. whether or not the QVF system is "realistic" or not). Bearing in mind that this closes the conceptual gap in the phenomenological description of Section 2.2, we will leave these technical and issues of interpretation aside.

Now, the direct accessibility of the atomic $\mathrm{CM}+\mathrm{R}$ structure is easily realized: the environment makes $\mathrm{CM}+$ $\mathrm{R}$ directly accessible to observation, while the atomic HA structure $\mathrm{e}+\mathrm{p}$ is "hidden" as its observation requires specific methods and procedures well known from the experimental investigation of the structure of matter.

The complexity of the theoretical modelling of atomic decays is an obstacle to a more elaborated operational use of these models. This is the reason that the phenomenological rule of Section 2.2 is still in wide use. Further progress in the foundations of the theory of open quantum systems can be expected to change this attitude.

\subsection{Quantum Mechanical Limit for the Hydrogen Atom}

For some higher energies the hydrogen atom breaks (ionizes) into a pair (e, p) where there are not the atomic (internal) bound states. But the above rule for the preferred structure remains the same: now every particle (e and p) polarizes the vacuum and separately induces the QVF-state changes [11]. In effect, QVF monitors every particle separately and distinguishes the well-known picture of the freely moving electrically charged particles, in reference to the structure $\mathrm{e}+\mathrm{p}$. This is the familiar picture from electrodynamics, both quantum and classical.

Again, according to Sections 2.1 and 3.2, for the separable state for $\mathrm{e}+\mathrm{p}$, it is in principle possible to observe entanglement for the alternative structure $\mathrm{CM}+\mathrm{R}$. However, as this is as yet speculative from the operational point of view, we will not herein elaborate on this possibility any further.

\section{Preferred Quantum Structures: An Outlook}

Sections 3 and 4 provide us with the following lessons.

First, for an isolated system, there is no argument and/or criterion or prescription to choose a preferred structure (decomposition into subsystems) of a composite system.

Second, not only operationally, but also from the more fundamental (e.g. the decoherence [11]-[14]) point of view: the choice of the preferred structure is made by the composite-system's environment.

Third, only when considering an atom as open system, we obtain answers to the issues of both "quantum jumps" (Section 2.2) and to the phenomenologically preferred structures (Section 3 and 4) of the chemical elements. Being physically incomparable and information-theoretically separated from the atomic $\mathrm{R}$ system, the atomic electron's "orbits" and "energies" cannot be inferred from the information provided solely by the R's quantum states $\psi_{n l m_{l}}\left(\rho_{R}\right)$.

\section{Discussion}

Modern quantum mechanics investigates quantum systems that interact with their environments. Being, in principle, undescribable by the Schrödinger law, such systems are termed "open". The theoretical basis is the socalled Theory of Open Systems, cf. e.g. [11]. Constant progress in our ability to comprehend open-systems dynamics opens new avenues in resolving some long-standing issues in the standard quantum theory of the isolated ("closed") quantum systems.

The exploration of quantum structures as we have expressed throughout this paper is relatively new to modern 
quantum theory, and while the structural variations are as old as quantum theory itself, only recent progress places it in proper context in modern quantum mechanics. To this end, the physical model of the Hydrogen Atom is paradigmatic. Not only does such structural analysis help to provide a deeper understanding, but also supports a more consistent and further simplified description of the Hydrogen Atom which, in turn, serves as a guide for deeper and more sophisticated descriptions of composite quantum systems.

\section{Conclusion}

Modern Quantum Theory extends and also deepens on our understanding of the quantum world. The lessons provided by the Theory of Open Quantum Systems rely heavily on the structure of composite systems. In this paper we make use of both, and offer a fresh look into the quantum mechanics of the hydrogen atom. We emphasize that modern quantum theory naturally and clearly answers two important questions: 1) how do the so called "quantum jumps" in atomic excitation and de-excitation occur? and 2) why does the classically and seemingly artificial "Center-of-Mass + relative degrees of freedom" structure appear as the primarily operable form in most of the experimental reality of atoms? and also that the hydrogen atom model itself is paradigmatic, and can be used as a guide in describing certain features of composite quantum systems.

\section{Acknowledgements}

JJD, MD and MA acknowledge financial support from Ministry of Education, Science and Technological Progress, Serbia, grant no 171028 and MD also from the ICTP-SEENET-MTP grant PRJ-09 "Strings and Cosmology" in frame of the SEENET-MTP Network.

\section{References}

[1] Dugić, M. (1999) What Is “System”: The Arguments from the Decoherence Theory. arXiv:quant-ph/9903037v1

[2] Zanardi, P. (2001) Virtual Quantum Subsystems. Physical Review Letters, 87, Article ID: 077901. http://dx.doi.org/10.1103/PhysRevLett.87.077901

[3] Dugić, M. and Jeknić, J. (2006) What Is “System”: Some Decoherence-Theory Arguments. International Journal of Theoretical Physics, 45, 2249-2259. http://dx.doi.org/10.1007/s10773-006-9186-0

[4] Ciancio, E., Giorda, P. and Zanardi, P. (2006) Mode Transformations and Entanglement Relativity in Bipartite Gaussian States. Physics Letters A, 354, 274-280. http://dx.doi.org/10.1016/j.physleta.2006.01.059

[5] Dugić, M. and Jeknić-Dugić, J. (2008) What Is "System": The Information-Theoretic Arguments. International Journal of Theoretical Physics, 47, 805-813. http://dx.doi.org/10.1007/s10773-007-9504-1

[6] De la Torre, A.C., et al. (2010) Ëntanglement for All Quantum States. European Journal of Physics, 31, $325-332$. http://dx.doi.org/10.1088/0143-0807/31/2/010

[7] Harshman, N.L. and Wickramasekara, S. (2007) Galilean and Dynamical Invariance of Entanglement in Particle Scattering. Physical Review Letters, 98, Article ID: 080406. http://dx.doi.org/10.1103/PhysRevLett.98.080406

[8] Jeknić-Dugić, J. and Dugić, M. (2008) Multiple System-Decomposition Method for Avoiding Quantum Decoherence. Chinese Physics Letters, 25, 371-374. http://dx.doi.org/10.1088/0256-307X/25/2/006

[9] Terra Cunha, M.O., Dunningham, J.A. and Vedral, V. (2007) Entanglement in Single-Particle Systems. Proceedings of the Royal Society A, 463, 2277-2286.

[10] Jeknić-Dugić, J., Dugić, M. and Francom, A. (2014) Quantum Structures of a Model-Universe: An Inconsistency with Everett Interpretation of Quantum Mechanics. International Journal of Theoretical Physics, 53, 169-180. http://dx.doi.org/10.1007/s10773-013-1794-x

[11] Breuer, H.-P. and Petruccione, F. (2002) The Theory of Open Quantum Systems. Clarendon Press, Oxford.

[12] Rivás, A. and Huelga, S.F. (2011) Open Quantum Systems: An Introduction. Springer Briefs in Physics, Springer, Berlin.

[13] Giulini, D., Joos, E., Kiefer, C., Kupsch, J., Stamatescu, I.-O. and Zeh, H.D. (1996) Decoherence and the Appearance of a Classical World in Quantum Theory. Springer, Berlin. http://dx.doi.org/10.1007/978-3-662-03263-3

[14] Zurek, W.H. (2003) Decoherence, Einselection, and the Quantum Origins of the Classical. Reviews of Modern Physics, 75, 715-775. http://dx.doi.org/10.1103/RevModPhys.75.715

[15] Fraser, G. (Ed.) (2006) The New Physics for the Twenty-First Century. Cambridge University Press, Cambridge. http://dx.doi.org/10.1017/CBO9781139644228 
[16] Tommasini, P., Timmermans, E. and Piza, A.F.R.D. (1998) The Hydrogen Atom as an Entangled Electron-Proton System. American Journal of Physics, 66, 881-885. http://dx.doi.org/10.1119/1.18977

[17] Li, Y., Bruder, C. and Sun, C.P. (2007) Generalized Stern-Gerlach Effect for Chiral Molecules. Physical Review Letters, 99, Article ID: 130403. http://dx.doi.org/10.1103/PhysRevLett.99.130403

[18] Gershnabel, E., Shapiro, M., Averbukh, I.Sh. (2011) Stern-Gerlach Deflection of Field-Free Aligned Paramagnetic Molecules. arXiv:1107.3916v1 [physics.chem-ph].

[19] Maeda, H., Norum, D.V.L. and Gallagher, T. F. (2005) Microwave Manipulation of an Atomic Electron in a Classical Orbit. Science, 307, 1757-1760. http://dx.doi.org/10.1126/science.1108470

[20] Rau, A.V., Dunningham, J.A. and Burnett, K. (2002) Measurement-Induced Relative-Position Localization through Entanglement. Science, 301, 1081-1084. http://dx.doi.org/10.1126/science.1084867

[21] Graham, R. and Miyazaki, M. (1996) Dynamical Localization of Atomic de Broglie Waves: The Influence of Spontaneous Emission. Physical Review A, 53, 2683-2693. http://dx.doi.org/10.1103/PhysRevA.53.2683

[22] Zhu, Z., Yu, H. and Lu, S. (2006) Spontaneous Excitation of an Accelerated Hydrogen Atom Coupled with Electromagnetic Vacuum Fluctuations. Physical Review D, 73 Article ID: 107501. http://dx.doi.org/10.1103/PhysRevD.73.107501 